\title{
EXPANSION OF FUNCTIONS IN SOLUTIONS OF FUNCTIONAL EQUATIONS*
}

LEONARD BRISTOW

1. Introduction. In analysis a number of functional equations have solutions of the form

$$
x^{r} \sum_{s=0}^{\infty} \alpha_{s, r} x^{s} .
$$

Examples are (a) linear differential equations with a regular singular point at the origin, (b) the Volterra homogeneous integral equation with a regular singularity, (c) the linear $q$-difference equation, (d) the Fuchsian equation of infinite order. There are many others including mixed $q$-difference and differential equations.

Consider the equation

$$
L(x, \lambda) \rightarrow y=0
$$

where $\lambda$ is a parameter and $L(x, \lambda)$ is an operator with the following property:

$$
L(x, \lambda) \rightarrow x^{p}=x^{p} f(x, p, \lambda)=x^{p} \sum_{\mu=0}^{\infty} f_{\mu}(p, \lambda) x^{\mu},
$$

the series converging for $|x| \leqq N<r$ for all values of $p$, which may be a complex number. The purpose of this paper is to consider under what conditions a set of values $\left\{\lambda_{m}\right\},(m=0,1,2, \cdots)$, can be determined so that for $\lambda=\lambda_{m}$ there will exist a solution of the form

$$
\begin{aligned}
y_{m+\sigma}(x) & =x^{m+\sigma} \sum_{s=0}^{\infty} \alpha_{s}^{(m+\sigma)} x^{s}=\sum_{s=0}^{\infty} \alpha_{s}^{(m+\sigma)} x^{m+\sigma+s} \\
& =x^{m+\sigma}\left\{\alpha_{0}^{(m+\sigma)} h_{m}(x)\right\}
\end{aligned}
$$

such that an arbitrary function $x^{\sigma} f(x), f(x)$ being analytic for $|x|<\rho$, can be expanded in a series

$$
x^{\sigma} f(x)=\sum_{m=0}^{\infty} a_{m} y_{m+\sigma}(x)
$$

which converges and represents the function in some region. For the

* Presented to the Society, October 29, 1938. 
sake of simplicity we will consider $\sigma=0$; the extension to $\sigma$ any number will be evident. In order to do this we will make use of the following theorem due to Mrs. Gertrude Stith Ketchum* which will be denoted by Theorem $\mathrm{K}$ :

TheOREM K. Consider the set of functions $g_{m}(x)=x^{m}\left\{1+h_{m}(x)\right\}$ where $h_{m}(0)=0$ and $h_{m}(x)$ is analytic for $|x|<r$. If there exists an $M_{N, m}$ such that $\left|h_{m}(x)\right| \leqq M_{N, m}$ for a given positive $N<r$ and $|x| \leqq N$, and if $\lim \sup M_{N, m}=K_{N}$ (finite), then any function analytic for $|x|<\rho$ has a unique uniformly convergent expansion $f(x)=\sum_{m=0}^{n=\infty} \alpha_{m} g_{m}(x)$ for $|x| \leqq R<G$, where

$$
G=\min \left\{p, \max _{N} N\left[1+K_{N}\right]^{-1}\right\},
$$

and the expansion converges absolutely for $|x|<G$.

We seek then to determine conditions under which the $y_{m}(x)$, $(m=0,1, \cdots)$, will satisfy the conditions of this theorem.

2. Sufficient conditions. Operating formally upon both sides of (4) $(\sigma=0)$ with the operator $L(x, \lambda)$ we get

$$
L(x, \lambda) \rightarrow y_{m}(x)=\sum_{s=0}^{\infty} \alpha_{s}^{(m)} x^{m+s} \sum_{\mu=0}^{\infty} f_{\mu}(m+s, \lambda) x^{\mu}=0 .
$$

Equating the coefficients of powers of $x$ to zero we get the following set of equations for the determination of the $\alpha_{s}{ }^{(m)},(s=0,1, \cdots)$ :

$$
\begin{aligned}
& \alpha_{0}^{(m)} f_{0}(m, \lambda)=0, \\
& \alpha_{1}^{(m)} f_{0}(m+1, \lambda)+\alpha_{0}^{(m)} f_{1}(m, \lambda)=0, \\
& . . \cdot . \cdot . \cdot . \cdot . \cdot . \cdot . \cdot \cdot, \\
& \alpha_{s+1}^{(m)} f_{0}(m+s+1, \lambda)+\alpha_{s}^{(m)} f_{1}(m+s, \lambda)+\cdots+\alpha_{0}^{(m)} f_{s+1}(m, \lambda)=0,
\end{aligned}
$$

If $\alpha_{0}{ }^{(m)} \neq 0$, then $f_{0}(m, \lambda)=0$ to give a solution of the desired form. Suppose that

$$
f_{0}\left(m, \lambda_{m}\right)=0, \quad m=0,1, \cdots,
$$

determines a set of characteristic values $\left\{\lambda_{m}\right\}$, and further suppose that

$$
f_{0}\left(p, \lambda_{m}\right) \neq 0, \quad m \neq p .
$$

\footnotetext{
* Transactions of this Society, vol. 40 (1935), pp. 208-224.
} 
The coefficients $\alpha_{s+1}^{(m)}$ can be determined for $s=0,1, \ldots$. Since $\alpha_{0}^{(m)}$ is arbitrary, we will choose it to be unity. By the method of Frobenius* we get the following set of inequalities:

$$
\begin{aligned}
\left|\alpha_{s+1}^{(m)}\right| & \leqq A_{s+1}^{(m)} \leqq A_{s}\left\{\frac{M_{N}\left(m+s, \lambda_{m}\right)+\left|f_{0}\left(m+s, \lambda_{m}\right)\right|}{\left|f_{0}\left(m+s+1, \lambda_{m}\right)\right|}\right\} \\
& =A_{s}^{(m)} P(m, s),
\end{aligned}
$$

where

$$
\begin{gathered}
A_{s+1}^{(m)}=\left\{\left|\alpha_{s}^{(m)}\right| M_{N}\left(m+s, \lambda_{m}\right)+\left|\alpha_{s-1}^{(m)}\right| M_{N}(m+s-1) N^{-1}+\cdots\right. \\
\left.+\left|\alpha_{0}^{(m)}\right| M_{N}(s) N^{-s}\right\}\left|f_{0}\left(m+s+1, \lambda_{m}\right)\right|^{-1}
\end{gathered}
$$

and $M_{N}\left(m+s, \lambda_{m}\right)$ are such that

$$
\left|\frac{d}{d x} f\left(x, m+s, \lambda_{m}\right)\right| \leqq M_{N}\left(m+s, \lambda_{m}\right) .
$$

It is evident that

$$
\left|h_{m}(x)\right| \leqq F_{m}(x)
$$

where

$$
F_{m}(x)=\sum_{s=1}^{\infty} A_{s}^{(m)}|x|^{s}
$$

Suppose

$$
\begin{aligned}
& \text { (a) } \limsup _{s \rightarrow \infty} P(m, s)=P(m), \\
& \text { (b) } \limsup _{m \rightarrow \infty} P(m)=p, \\
& \text { (c) } \limsup _{m \rightarrow \infty} P(m, s)=Q(s), \\
& \text { (d) } \limsup _{s \rightarrow \infty} Q(s)=q .
\end{aligned}
$$

Let $R$ be the smallest of $(P(m))^{-1},(m=0,1, \cdots), p^{-1}, q^{-1}$, and $N$. Then

$$
\limsup _{s \rightarrow \infty} A_{s+1}^{(m)} / A_{s}^{(m)} \leqq P(m),
$$

and (14) converges for $|x|<R$. Since $N$ is at our choice, let $N$ be less than $R$. We have also

* Journal für die reine und angewandte Mathematik, vol. 76 (1873), p. 214. 


$$
A_{s+1}^{(m)} \leqq \prod_{i=0}^{s} P(m, i)
$$

hence

$$
\limsup _{m \rightarrow \infty} A_{s+1}^{(m)} \leqq \prod_{i=0}^{s} Q(i)=A_{s+1}
$$

and

$$
\limsup _{s \rightarrow \infty} A_{s+1} / A_{s} \leqq q
$$

Then the series

$$
F(x)=\sum_{s=0}^{\infty} A_{s}|x|^{s}
$$

converges for $|x| \leqq N<R$.

Let $M_{N}^{(m)}$ be such that $\left|h_{m}(x)\right| \leqq F_{m}(x) \leqq M_{N}^{(m)}$ and $M_{N}$ such that $F(x) \leqq M_{N} ;$ then

$$
\limsup _{m \rightarrow \infty} M_{N}^{(m)}=K_{N} \leqq M_{N}
$$

The conditions of Theorem $\mathrm{K}$ are satisfied and we may state the following theorem:

THEOREM. If we have a functional equation with an operator having the property (3), if there exists a set of values fulfilling conditions (8) and (9), and if conditions (15) are satisfied, then there exists a unique expansion of the form

$$
f(x)=\sum_{m=0}^{\infty} a_{m} y_{m}(x),
$$

where $f(x)$ is analytic for $|x|<\rho$, which will converge uniformly for $|x| \leqq R<G$, where

$$
G=\min \left\{\max _{N} N\left(1+K_{N}\right)^{-1}\right\} .
$$

The expansion converges and represents the function for $|x|<G$.

3. Examples. Suppose we have the equation

$$
\begin{aligned}
\sum_{j=0}^{n} P_{j, 0}(x, \lambda) \delta^{n-j} y(x) & +\sum_{i=1}^{r} \lambda^{i} \sum_{j=0}^{m} P_{j, i}(x, \lambda) \delta^{m-j} y(x) \\
& +\int_{0}^{x} g(x, t, \lambda) y(t) d t=0,
\end{aligned}
$$


where

$$
\begin{aligned}
P_{0,0}(x) & \equiv 1,\left|\frac{d}{d x} P_{j, i}(x, \lambda)\right| \leqq M_{N, N}^{(j, i)},|x| \leqq N<r,|\lambda|>N, \\
g(x, t, \lambda) & =\sum_{i=0}^{\infty} \sum_{j=0}^{\infty} b_{i j}(\lambda) x^{i} t^{j}, \\
G(x, p, \lambda) & =\sum_{i=0}^{\infty} \sum_{j=0}^{\infty} b_{i j}(\lambda) \frac{i+j+1}{i+p+1} x^{i+j}, p \text { an integer, }
\end{aligned}
$$

and

$$
|G(x, p, \lambda)| \leqq M_{N, N}, \quad|x| \leqq N<r, \quad|\lambda|>N .
$$

The function $\delta^{s} y(x)$ is either $x^{s} d^{s} y / d x^{s}, y\left(q_{s} x\right)$ with $\left|q_{n}\right|>1$ and $\left|q_{n}\right|>\left|q_{n-i}\right|,(i=1,2, \cdots, n)$, or $y\left(q^{s} x\right)$ with $|q|>1$. The function $f_{0}(m, \lambda)$ will be a polynomial of degree $r$ in $\lambda$ and of degree $n$ in either $q^{m}$ or $m$, or a polynomial of degree $m$ in $q_{j},(j=0,1, \cdots, n)$. The conditions of the theorem can then be shown to be satisfied, and the expansion of an arbitrary function follows. Consider the case for which $r=1, m=0$, and $P_{n, i}(x, \lambda), g(x, t, \lambda)$ are independent of $\lambda$. If $\left\{\lambda_{m}\right\},(m=0,1, \cdots)$, is the set of characteristic values and $y_{m}(x)$ are the corresponding functions, then the solution of the nonhomogeneous equation

$$
\begin{aligned}
\delta^{n} y(x) & +P_{1}(x) \delta^{n-1} y(x)+\cdots+P_{0}(x) y(x) \\
& +\int_{0}^{x} g(x, t) y(t) d t+\lambda y(x)=f(x),
\end{aligned}
$$

where $f(x)$ is analytic for $|x|<\rho$, has a solution of the form

$$
y(x)=\sum_{m=0}^{\infty} \frac{a_{m}}{\lambda-\lambda_{m}} y_{m}(x), \quad \lambda \neq \lambda_{m},
$$

where $f(x)=\sum_{m=0}^{\infty} a_{m} y_{m}(x)$. This is easily verified by substitution. If $\lambda=\lambda_{p}$ and $f(x)=x^{p+1} F(x)$, then the solution is of the form

$$
y(x)=\sum_{m=p+1}^{\infty} \frac{a_{m}}{\lambda_{p}-\lambda_{m}} y_{m}(x) .
$$

Consider the equation

$$
\sum_{n=0}^{\infty} \frac{A_{n}(x, \lambda)}{n !}\left(x \frac{d y}{d x}\right)^{n}+\lambda y(x)=0,
$$

where 


$$
\begin{aligned}
\frac{d}{d x} A_{n}(x, \lambda) \equiv 0, \quad n>n^{\prime}, \quad\left|\frac{d}{d x} A_{n}(x, \lambda)\right| & \leqq M_{N, N}^{(n)}, \\
|x| & <N<r, \quad|\lambda|>N,
\end{aligned}
$$

$A_{n}(0, \lambda) \neq 0,\left(n>n^{\prime}\right), A_{n}(0, \lambda)=a_{n}$ independent of $\lambda$, and

$$
\begin{aligned}
& \left(x \frac{d y}{d x}\right)^{1}=x \frac{d y}{d x}, \quad\left(x \frac{d y}{d x}\right)^{2}=x \frac{d}{d x}\left\{x \frac{d y}{d x}\right\}, \\
& \left(x \frac{d y}{d x}\right)^{p}=x \frac{d}{d x}\left\{\left(x \frac{d y}{d x}\right)^{p-1}\right\} .
\end{aligned}
$$

Then we obtain the relations

$$
f_{0}(m, \lambda)=\sum_{n=0}^{\infty} \frac{a_{n} m^{n}}{n !}+\lambda=0, \quad \lambda=-\sum_{n=0}^{\infty} \frac{a_{n} m^{n}}{n !}=-f(m) .
$$

If $a_{n}=1, \lambda_{m}=-e^{m}$.

This equation and others in which $\lambda_{m}$ has the properties

$$
\begin{aligned}
& \limsup _{m \rightarrow \infty} \frac{|P(m+s)|}{\left|\lambda_{m+s+1}-\lambda_{m}\right|}=Q(s), \\
& \limsup _{s \rightarrow \infty} Q(s)=q \text {, } \\
& \limsup _{s \rightarrow \infty} \frac{|P(m+s)|}{\left|\lambda_{m+s+1}-\lambda_{m}\right|}=\bar{P}(m), \\
& \limsup _{m \rightarrow \infty} \bar{P}(m)=p \text {, }
\end{aligned}
$$

$P(m+s)$ being a polynomial in $m+s$, will satisfy the conditions of the theorem, and the expansion follows.

The generalized Fuchsian equation

$$
\sum_{n=0}^{\infty} x^{n} \frac{A_{n}(x, \lambda)}{n !} \frac{d^{n} y}{d x^{n}}+\lambda y(x)=0
$$

is similar to the above except for the fact that the $\lambda_{m}$ are given by Newton series.

UNIVERSITY OF ILLINOIS 